DOI: http://dx.doi.org/10.54085/ap.covid19.2021.10.2.1
Annals of Phytomedicine: An International Journal
http://www.ukaazpublications.com/publications/index.php
Print ISSN : 2278-9839 Online ISSN : 2393-9885

\title{
Phytochemicals and COVID-19
}

\section{Prafulla Parikh}

Advanced Centre for Treatment Research and Education in Cancer (ACTREC), Tata Memorial Centre, Homi Bhabha National Institute (HBNI), Navi Mumbai-400012, India

\author{
Article Info \\ Article history : Received 10 October 2021, Revised 17 November 2021, Accepted 19 November 2021 , Published Online 30 December 2021
}

\section{Introduction}

COVID-19 which originated from Wuhan in China in 2019 was declared as pandemic by the WHO, has affected more than 200 countries around the world and millions of human lives were lost due to COVID-19 infection. After coronavirus infection, patient may suffer from asymptomatic infection to life threatening various organ dysfunction leading to mortality. In some individuals, long term sequel has resulted in various long term debilitating illnesses and data are still evolving for long term sequel. COVID-19 is the deadliest infection of this century. Presently, fear of third wave by Omicron variant of corona is gripping the world. Booster doses of vaccines have been announced hurriedly but efficacy against Omicron is doubtful. Data about morbidity and mortality due to Omicron variant is insufficient and evolving. Preventive measures (mask, social distancing) are more effective in absence of definitive treatment options and are being reinforced time and again.

COVID-19 is a challenge to science as even after more than 24 months of identification of virus, definitive treatment modalities are unavailable. Virus is mutating frequently and various mutants of corona have different pattern of transmissibility, severity and ability to evade vaccines. More than 3000 randomized controlled trials (according to www.covid-nma.com) are going on for finding the treatment of COVID-19, out of which still more than 1500 trials are recruiting participants. Definitive pre-exposure and post exposure prophylaxis is also unclear.

Morbidity and mortality due to various infective illness has increased in last decade and its main puzzle for scientists to find effective antimicrobial agents. Resistance to various antimicrobials is worrisome. COVID-19 pandemic came suddenly and many antivirals, antimalarials, antibiotics were repurposed to find therapy for COVID-19.

Phytochemicals from plants is a valuable field of research to explore and re-establish its importance. Human race exists long before the invention of allopathy and medicinal plants were the main source of various therapeutic drugs. Since ancient times various plants have been used for treatment of various febrile illnesses also due to their strong therapeutic effects. With the change in living style, environment, the dietary pattern disease presentation has changed. Communicable, infectious diseases are happening more frequently and becoming an important cause of morbidity and mortality. With changing disease pattern, spectrum of long term and short term persistent symptoms is also changing. Resistance to antibiotics,

Copyright () 2021 Ukaaz Publications. All rights reserved.

Email: ukaaz@yahoo.com; Website: www.ukaazpublications.com antivirals and antiparasitic drugs is puzzling to scientists and world is desperately hunting for more better alternatives to treat these infections.Though, phytochemicals are time tested proven therapeutic agents, repurposing of these important substances is vital to find therapy with maximum efficacy and minimal side effects. Holistic approach to various infections is being welcomed more nowadays which involves usage of immunity boosters with antimicrobial agents. Phytochemicals based development of phytomedicine is one of hopes world is looking for. Phytochemicals exist in our food and they play important role in maintaining physiological balance of the body. Constant supply of these nutrients is vital that may be the reason for prescribing specific diet during illness. Careful selection of food items and medicinal plants is important to re-establish strong immunity to fight the microbial agents.

Phytochemicals also can be repurposed during this pandemic based on previous evidences. Major advantage of phytochemicals is that they have plethora of effects. Phytochemicals play an important role as immunity booster, antiviral, antioxidants, etc. For example, amla is not only giving vitamin $\mathrm{C}$ but it also has antioxidant, antidiabetic and many other effects. Various phytochemicals have synergistic effects and such synergistic combinations need evaluation for maximum therapeutic benefits.

\section{Non-communicable diseases and covid}

Phytomedicine is becoming popular for management of life style disorders and non-communicable diseases, e.g., malignancy, diabetes, obesity, hypertension. Non-communicable diseases play important role in management of communicable diseases (bacterial, viral) and other infectious diseases. It has mirrored in COVID-19 management too. COVID-19-outcome was affected by these non-communicable diseases. Co-morbidities were responsible for increased mortality during COVID-19. Individuals having pre-existing noncommunicable diseases are more prone to COVID-19 complications. Even after COVID-19 infection, many patients have developed diabetes mellitus type 2 , hypertension which may be diagnosed as long term sequel of COVID-19 or side effect of COVID-19 therapy, e.g, due to steroids.

WHO recommends use of local medicinal plants in treatment of both communicable and non-communicable diseases. Knowingly or unknowingly phyotchemicals are part of our day-to-day diet. Seasonal food, fruits are also important source of phytochemicals. 
Dose, duration of phytochemicals when used for therapeutic purposes is high and need standardization based on scientific evidences. For non-communicable diseases, curcumin is leading at present. Trials in diabetic patients, malignancy patients, patients with metabolic syndrome are going on. In neurodegenerative disorders also various herbs are being tested on large scale.

For communicable diseases since last many years new group of antibiotics has not been discovered. Many antibiotics, antivirals, antiparasitic drugs have been repurposed. Vacuum in holistic approach of treatment of infectious diseases is being felt by international scientific community.

Many medicinal plants have excellent antibacterial, antiviral activity with minimal side effects. These medicinal plants have synergistic activity with other herbs and they are more effective when taken into combination.

\section{Phytochemicals as immunity booster, immunomodulator and medicine}

An individual's immune system plays vital role in fight against infectious agents. COVID-19 also is no exception. Elderly with weak immune system, persons on immunosuppressive agents got severely affected. Immune system is the target for various therapeutic drugs now. Immunomodulators are being investigated with various degrees of success but meta analysis have not shown any promising results.

Relation between phytochemicals and immune system is multidimensional. Vitamin $\mathrm{C}$ and zinc emerged as the most important immunity booster during this deadly pandemic.

Plant based treatment for infectious diseases is gaining attraction worldwide as it is supposed to have less side effects and probably have multiple effects as anti-infectious agents, immunity booster, antioxidant, etc. ( Yadav and Kaushik, 2021)

Phytochemicals have synergistic effect and using the plant extract is more useful than isolating single active ingredient and using it. Bioavailability of crude extract also depends on various factors, e.g, age, gender, environmental factors, method of preparation, food combinations, etc. Bioavailability of curcumin in turmeric increases when taken with piperine and in fat soluble medium. Increasing bioavailability during acute illness and during day to day consumption is very important. Decreasing duration of acute illness with minimal long term sequel is very important target during treatment of febrile illness.

More high quality clinically relevant trials can shed light on the full potential of phytochemicals as novel antiviral agents with better delivery system to offer maximum therapeutic benefit.

The lack of COVID-19-oriented conventional therapeutic agents (vaccines, antibiotics) enforced the use of supportive therapies primarily based on the agents of natural origin. Extracts from natural products are a rich source of active compounds that can be used against coronavirus as a solution of sorts. The wide range of natural substances present in herbal extracts, which are underrated in conventional medicine, may constitute almost inexhaustible source of medicaments (Chojnackaa et al., 2020).
There is potential for the use of plant-derived polyphenols as functional foods and pharmaceutical preparations. The raw materials for their preparation, namely; herbs and consumable plants, are approved for human consumption worldwide, this makes the method of implementation of such preparations fast (Chojnackaa et al., 2020).

After the outbreak of COVID-19, in silico studies published reported the possibility of utilizing phytochemicals to treat or prevent COVID-19. Binding affinity studies suggested good possibilities of using phytochemicals. Because of differences in the type of molecular structures that they showed affinity mechanisms of action would be different. Gingerol, a phenolic compound in ginger (Zingiber officinale), showed high affinity to a SARS-CoV-2 main protease $5 \mathrm{R} 7 \mathrm{Y}(-15.7591 \mathrm{~kJ} / \mathrm{mol})$. It also shows binding affinities to RNA binding proteins $6 \mathrm{~W} 4 \mathrm{~B}(-11.4082 \mathrm{~kJ} / \mathrm{mol})$ and $6 \mathrm{VSB}$ (Rathinavel et al., 2020).

For centuries, phytochemicals have also been known to have effects on moods and physiological conditions. However, scientific studies on these aspects have started recently. Multiple routes are possibly involved to cause a neuroendocrinological change which reflects in mood changes and physiological conditions and through absorption to the skin and digestive system. Linalool, is one of the major chemical components of lavender essential oil. Exposure to the odor of linalool induced significant analgesic and anxiolytic effects in mice (Harada et al., 2018; Tashiro et al., 2016). Also, an exposure to lavender, which contains linalool, reduced stress levels and anxiety, enhanced positive moods, increased a relaxing mood, and increased the percentage of deep slow wave sleep. Adult human subjects who inhaled (R) (-) - linalool showed reduced heart rate and produced a calm and vigorous mood (Kuroda et al., 2005; Sowndhararajan et al., 2016).

Precision medicine is an approach based on the disease treatment and prevention considering individual variability. If, the biological properties of different group of phytochemicals could be well established, these could be utilised in the precision medicine.

One of the major symptoms of COVID-19 is inflammation. Various phytochemicals are well known for their anti-inflammatory properties. These phytochemical based therapies could be useful in facilitating recoveries from COVID-19 induced anosmia and ageusia (Koyama et al., 2021).

The loss of chemical senses, i.e., smell and taste is one of the unique symptoms of the COVID-19. One of the methods used in facilitating recovery of the olfactory sense is smell training. This therapy uses essential oils of lemon, rose, clove, and eucalyptus. Scientific studies have shown that essential oils improve recovery. However, there may be better combinations for facilitating recovery (Koyama et al., 2021).

Angiotensin-converting enzyme 2 (ACE2) is a receptor. ACE2 is responsible for the cellular entry of SARS-CoV-2. Transmembrane protease serine 2 (TMPRSS2), a protease, facilitates viral entry into the host cells. Essential oils that have both the function of suppressing the secretion of cytokines as well as suppressing the expression of ACE2 and TMPRSS 2 could be one of the promising methods to prevent and treat COVID-19. Citronellol and geraniol exposure (at a concentration that is not cytotoxic) to a human colorectal adenocarcinoma cell line (HT-29) significantly downregulated the expression of ACE2 and TMPRSS2. This suggests that 
they may become one of the candidates for anti-COVID-19 treatments.

Opportunities to utilize phytochemicals are not limited to as supplemental treatments in clinical settings. It might be possible to develop various agents which will help prevent contraction of the virus, such as a coating on masks, a spray for surfaces, diffusers to reduce virus in airborne particles, and so on by modifying the concentrations and chemical stability (Koyama et al., 2021). Also, a possible way to accelerate use of phytochemicals would be to start using phytochemicals with no side effects that are food grade nutrients, and used in combinations instead of as a single additive in order to enhance the chances that it works, and meanwhile proceed on testing other candidates.

New processing and formulation technologies might help optimize the solubility of antiviral phytochemicals. In addition to that, optimization of their delivery strategies and therapeutic activities would be beneficial to adapt them as antiviral functional foods and drugs (Lin et al., 2014; Zhong et al., 2012). Bioinformatic studies of the phytopharmacophores structures can can lead to designing new antiviral drugs (Ul Qamar et al., 2020).

It would be worthy to conclude that phytochemicals may serve as a springboard for future clinical trials, evaluating the efficacy of available phytochemicals/supplements for discovery of therapeutics.

Phytomedicine is a multifaceted subject with areas including ethnopharmacology, botany, biochemistry, pharmacology, ethnobotany, molecular biology, biostatistics, taxonomy, phytochemistry, etc. Researchers from this area seek a platform where their findings could be published after through peer-review. Annals of Phytomedicine: An International Journal (both online and print version), is a highly accepted journal to publish good quality research findings in the area of medicinal plants. In this area, this journal is well recognised for its timely publishing of volume and critical review of the research articles.

\section{Conflict of interest}

The author declares no conflicts of interest relevant to this article.

\section{References}

Chojnackaa, K.; Witek-Krowiaka, A.; Skrzypczaka, D.; . Mikulaa, K. and M³narz, P. (2020). Phytochemicals containing biologically active polyphenols as an effective agent against COVID-19-inducing coronavirus. Journal of Functional Foods, 73:1041-1046.

Harada, H.; Kashiwadani, H.; Kanmura, Y. and Kuwaki, T. (2018). Linalool odor-induced anxiolytic effects in mice. Frontiers in Behavioral Neuroscience, 12:241.

Koyama, S.; Kondo, K.; Ueha, R.; Kashiwadani, H. and Heinbockel, T. (2021). Possible use of phytochemicals for recovery from COVID-19induced anosmia and ageusia. International Journal of Molecular Science, 22:8912.

Kumar, K.J.S.; Vani, M.G.; Wang, C.S.; Chen, C.C.; Chen, Y.C.; Lu, L.P.; Huang, C.H.; Lai, C.S. and Wang, S.Y. (2009). Geranium and lemon essential oils and their active compounds downregulate angiotensinconverting enzyme 2 (ACE2), a SARS-CoV-2 spike receptorbinding domain, in epithelial cells. Plants, 9:770.

Kuroda, K.; Inoue, N.; Ito, Y.; Kubota, K.; Sugimoto,A.; Kakuda, T. and Fushiki, T. (2005). Sedative effects of the jasmine tea odor and (R)-(-)linalool, one of its major odor components, on autonomic nerve activity and mood states. The European Journal of Applied Physiology, 95:107-114.

Lin, L.T.; Hsu, W.C.; and Lin, C.C. (2014). Antiviral natural products and herbal medicines. Journal of Traditional and Complementary Medicine, 4:24-35.

Rathinavel, T.; Palanisamy, M.; Palanisamy, S.; Subramanian, A. and Thangaswamy, S. (2020). Phytochemical 6-gingerol: A promising drug of choice for COVID-19. International Journal of Applied Science and Engineering, 6:1482-1489.

Sowndhararajan, K. and Kim, S. (2016). Influence of fragrances on human psychophysiological activity: with special reference to human electroencephalographic response. Scientia Pharmaceutica, 84: 724-751.

Tashiro, S.; Yamaguchi, R.; Ishikawa, S.; Sakurai, T.; Kajiya, K.; Kanmura, Y.; Kuwaki, T. and Kashiwadani, H. (2016). Odour-induced analgesia mediated by hypothalamic orexin neurons in mice. Scientific Reports, 6:37-129.

UI Qamar, M.T.; Alqahtani, S. M.; Alamri, M. A. and Chen, L.-L. (2020). Structural basis of SARS-CoV-2 3CLpro and anti-COVID-19 drug discovery from medicinal plants. Journal of Pharmaceutical Analysis, 10:313-319

Yadav, V.K. and Kaushik, P. (2021). Phytochemicals against COVID-19 and a gap in clinical investigations: An outlook. Indian Journal of Biochemistry and Biophysics, 58:403-407

Zhong, Y.; Ma, C. M. and Shahidi, F. (2012). Antioxidant and antiviral activities of lipophilic epigallocatechin gallate (EGCG) derivatives. Journal of Functional Foods, 4:87-93. 


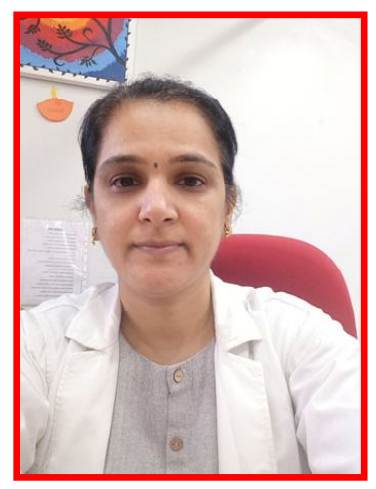

Dr. Prafulla Parikh

Professor of Internal Medicine at Clinical Research Centre, Advanced Centre for Treatment Research and Education in Cancer (ACTREC), Tata Memorial Centre, Homi Bhabha National Institute (HBNI), Navi Mumbai-400012, India

\section{Biography}

She undertook her MBBS, MD (Internal Medicine) training from B.J. Medical College, Ahmedabad. She is a passionate physician with extensive experience (more than 16 years) in internal medicine. She has worked at prestigious institute like Pramukh Swami Medical College, Karamsad as an Assistant Professor before joining Tata Memorial Centre, Mumbai. As a clinician, she is very agile, quick in making correct decisions and efficient in handling patients. She is ready to put in significant efforts in developing new frontiers in Internal medicine. She has been actively participating in patient educational activities of Diabetes club.

Dr. Prafulla has many innovative ideas for teaching Undergraduate and Postgraduate students in the department. She is a strict disciplinarian and expects to see the learning curve of the students growing well. She keeps herself abreast with latest in internal medicine by attending number of conferences in the super specialities of Medicine. On research front, she is working on many phase II and phase III trials. A firm believer in holistic approach for patient treatment, she feels Indian medicinal plants are treasure to the world and must be explored to the fullest in our daily lives as well as for treating communicable infectious diseases and non-communicable diseases. Dr. Parikh has taught juniors, senior residents and nursing staff and students. She has published several research papers in prestigious scientific national/international journals that cater to the fields of medicine, science and ayurveda. Dr. Prafulla is cost conscious professional ready to serve the humanity at large. 